\title{
ON PERIODIC SOLUTIONS OF NONLINEAR HYPERBOLIC EQUATIONS AND THE CALCULUS OF VARIATIONS
}

\author{
BY MELVYN S. BERGER
}

Communicated by Wolfgang Wasow, January 10, 1969

Let $G$ be a bounded domain in $R^{N}$ with boundary $\partial G$. Then the system (for $p(x)$ a strictly positive $C^{\prime}(\bar{G})$ function)

$$
\begin{aligned}
p(x) u_{t t}-\Delta u & =0 \quad(\text { in } G), \\
u / \partial G & =0,
\end{aligned}
$$

has a countably infinite number of distinct periodic solutions (i.e. "normal modes"). In this note we shall show that the same conclusion can be established for the nonlinear system

$$
\begin{aligned}
p(x) u_{t t}-\Delta u+f(x, u) & =0, \\
u / \partial G & =0,
\end{aligned}
$$

under certain restrictions on the functions $f(x, u)$ and $p(x)$. (Throughout we assume $f(x, 0) \equiv 0$, so that $u(x, t) \equiv 0$ satisfies (2).) Furthermore similar results can be obtained for higher order systems in which the Laplace operator $\Delta$ is replaced by a strongly elliptic operator of order $2 m$ and the boundary conditions are suitably altered (such systems occur in the theory of elastic vibrations).

Our proofs are based on approximating the system (2) by a Hamiltonian system of ordinary differential equations, as in [4]. The periodic solutions of the associated Hamiltonian systems are then investigated by the methods of the calculus of variations in the large, as studied by the author in [1]. Periodic solutions of the original system (2) are then obtained by taking limits. Previous mathematical studies of periodic solutions of (2) (e.g. [2], [3], [5]) have been primarily perturbation results and have not considered the totality of periodic solutions of (2).

1. Preliminaries. Let $x$ denote a point in $G$ and $W_{1,2}\left(G_{T}\right)$ denote the Sobolev space of functions $u(x, t), T$-periodic in $t$, which are square integrable and possess square integrable derivatives over $G \times[0, T]$. By $\dot{W}_{1,2}\left(G_{T}\right)$ we denote the subspace of $W_{1,2}\left(G_{T}\right)$ consisting of functions which vanish on $\partial G$ (in the generalized sense). $\dot{W}_{1,2}\left(G_{T}\right)$ is a Hilbert space with respect to the inner product 


$$
(u, v)_{1,2}^{T}=\int_{0}^{T} \int_{G}\left\{u_{t} v_{t}+\operatorname{grad} u \cdot \operatorname{grad} v\right\} .
$$

By a $T$-periodic weak solution of (2), we understand a function $u(x, t) \in \dot{W}_{1,2}\left(G_{T}\right)$ which satisfies the following integral identity for all $\phi \in \dot{W}_{1,2}\left(G_{T}\right):$

$$
0=\int_{0}^{T} \int_{G}\left\{u_{t} \phi_{t}-\operatorname{grad} u \cdot \operatorname{grad} \phi-f(x, u) \phi\right\} .
$$

Henceforth we shall study $T$-periodic weak solutions $u(x, t) \not \equiv 0$.

2. Statement of results. First we discuss the existence of a global one-parameter family of periodic solutions of (2) whose frequency corresponds roughly to the lowest eigenvalue $\lambda_{1}^{2}$ of $\Delta$ on $G$. To this end, we assume that (i) $f(x, u)$ satisfies the following growth condition:

$$
|f(x, u)| \leqq K_{0}|u|, \text { for }|u| \text { sufficiently large }
$$

and $F(x, u) \leqq K_{1} f(x, u) u$ where $F(x, u)=\int_{0}^{u} f(x, t) d t$ and $K_{0}, K_{1}$ are positive constants independent of $u$ and $x$ (ii) $\left(\mathrm{I}_{i}\right) \lambda_{j} / \lambda_{i}=$ integer is satisfied for at most finitely many indices $j$, where $\lambda_{j}^{2}$ denote the eigenvalues of $\Delta$ on $G$ with respect to $p(x)$.

THEOREM 1. Let $f(x, t)$ be a locally Lipschitz continuous function, odd in $t$, satisfying the conditions $\left(^{*}\right)$ and $\left(\mathrm{I}_{1}\right)$ and such that for all real $t$ and $x \in G, t f(x, t) \geqq 0$. Then the system (2) has a one-parameter family of distinct $T_{1}(R)$-periodic weak solutions $\tilde{u}_{1}(R)$ where the parameter $R$ is sufficiently small, provided $f(x, t)=o(t)$ for small $t$. In addition, $R$ and $T_{1}(R)$ are related by

$$
2 \pi R=T_{1}(R) \int_{0}^{T_{1}(R)} \int_{G}\left(\frac{\partial \tilde{u}_{1}(R)}{\partial t}\right)^{2} .
$$

Furthermore as $R \rightarrow 0, T_{1}(R) \rightarrow 2 \pi \lambda_{1}^{-1}$, where $\lambda_{1}^{2}$ is the smallest eigenvalue of $\Delta$.

The next result concerns the existence of an infinite number of distinct one-parameter families of periodic solutions of (2), whose frequencies correspond roughly to the other eigenvalues of $\Delta$ on $G$.

THEOREM 2. Let $f(x, t)$ be a locally Lipschitz function of $x$ and $t$, odd in $t$, satisfying the conditions $\left(^{*}\right)$ and $\left(\mathrm{I}_{\mathrm{i}}\right)$, and such that $f(x, t)=o(t)$ for small $t$. Then the system (2) has, for sufficiently small $R$, an infinite 
number of distinct one-parameter families of $T_{i}(R)$-periodic weak solutions $u_{i}(R)(i=1,2, \cdots)$, where $R$ is defined by (4). Furthermore as $R \rightarrow 0, T_{i}(R) \rightarrow 2 \pi \lambda_{i}^{-1}$ where $\lambda_{i}^{2}$ denote the eigenvalues of $\Delta$ with respect to $p(x)$ ordered by magnitude.

3. Outline of proofs. Denote the eigenvalues (ordered by magnitude) and eigenfunctions of $\Delta$ with respect to $p(x)$ by $\lambda_{i}^{2}$ and $u_{i}(x)$, respectively. Then approximate weak solutions of (2) by functions of the form $\tilde{u}_{n}=\sum_{i=1}^{n} q_{i}^{(n)}(t) u_{i}(x)$, where $q_{i}^{(n)}(t)$ are functions to be determined. Substituting $\tilde{u}_{n}$ in (2) we find the following approximate equations for $q_{i}^{(n)}(t)$.

$$
\ddot{q}_{i}^{(n)}+\lambda_{i}^{2} q_{i}^{(n)}+\int_{G} p f\left(x, \sum_{j=1}^{n} q_{j}^{(n)} u_{j}\right) u_{i}=0 \quad(i=1, \cdots, n) .
$$

Setting $t=\sigma s$, we consider $2 \pi$-periodic solutions of the system

$$
\ddot{q}_{i}^{(n)}+\sigma^{2}\left[\lambda_{i}^{2} q_{i}^{(n)}+\int_{G} p f\left(x, \sum_{j=1}^{n} q_{j}^{(n)} u_{j}\right) u_{i}\right]=0(i=1, \cdots, n) .
$$

The $2 \pi$ periodic solutions of (6) can be regarded as critical points of the functional

$$
\mathcal{S}_{n}\left(q^{(n)}\right)=\int_{0}^{2 \pi}\left\{\sum_{i=1}^{n} \lambda_{i}^{2}\left(q_{i}^{(n)}\right)^{2}+\int_{G} p 2 F\left(x, \sum_{i=1}^{n} q_{i}^{(n)} u_{i}\right)\right\}
$$

over the admissible class of odd, $2 \pi$ periodic $n$-vector functions $q^{(n)}$ $=\left(q_{1}^{(n)}, q_{2}^{(n)}, \cdots, q_{n}^{(n)}\right)$ such that $\int_{0}^{2 \pi}\left(\dot{q}^{(n)}\right)^{2}=R$, a positive constant. We compare these critical points with the critical points of the "linearized" problem $V_{R}(n)$ : i.e. the critical points of the functional

$$
Q_{n}\left(q^{(n)}\right)=\int_{0}^{2 \pi} \sum_{i=1}^{n} \lambda_{i}^{2}\left(q_{i}^{(n)}\right)^{2}
$$

over the same admissible class of functions as above. The critical values of $V_{R}(n)$ are proportional to $\lambda_{i}^{2} / K^{2},(i=1,2, \cdots, n), K$ an integer. In the following we order these critical values in decreasing order of magnitude and denote the critical value proportional to $\lambda_{i}^{2}$ as the $n(i)$ th number in this ordering (with multiplicities included).

The proofs make use of

(i) the techniques of the Ljusternik-Schnirelmann theory of critical points on Hilbert manifolds for fixed $n$, and

(ii) a limiting selection procedure as $n \rightarrow \infty$.

Consider the set of continuous odd $2 \pi$ periodic $n$-vector functions 
$q^{(n)}(s)$ which possess a square integrable generalized derivative. This set forms a Hilbert space $H^{n}$ with norm

$$
\left\|q^{(n)}\right\|^{2}=\sum_{i=1}^{n} \int_{0}^{2 \pi} \dot{q}_{i}^{2}(s) d s .
$$

In $H^{n}$ we identify the antipodal points on the sphere $S^{n}(R)$ $=\left\{q^{(n)}\left\|q^{(n)}\right\|^{2}=R\right\}$ to obtain the infinite dimensional real projective space $P_{R}^{\infty}\left(H^{n}\right)$, and by virtue of the evenness of $\mathcal{S}_{n}\left(q^{(n)}\right)$, we consider $\mathcal{G}_{n}\left(q^{(n)}\right)$ as defined on $P_{R}^{\infty}\left(H^{n}\right)$. To prove Theorem 1, we consider the variational problem

$$
V(n, 1): c_{n(1)}(R)=\sup _{[W]_{n}(1)} \min _{W} \mathcal{G}_{n}\left(q^{(n)}\right)
$$

where

$$
[W]_{n(1)}=\left\{W \mid W \subset P_{R}^{\infty}\left(H^{n}\right), \operatorname{cat}\left(W, P_{R}^{\infty}\left(H^{n}\right)\right) \geqq n(1)\right\} .
$$

The problem $V(n, 1)$ has a solution $q_{i}^{(n)}(s)$ which is a critical point of $\oint_{n}\left(q^{(n)}\right)$ and so satisfies (6) for some $\sigma_{n(1)}(R)$, thus giving rise to a $2 \pi \sigma_{n(1)}(R)$ periodic solution of (5). As $R$ runs through small positive values, one-parameter families of periodic solutions of (5) are generated; as in [1]. Now as $n \rightarrow \infty$ for fixed $R$, the sequences $\left\{c_{n(1)}(R)\right\}$, $\left\{\sigma_{n(1)}(R)\right\}$ and $\left\{\left\|\sum_{i=1}^{n} u_{i}(x) q_{i}^{(n)}(s)\right\|_{1,2}^{2 \pi}\right\}$ are uniformly bounded, so that (after suitable reindexing) there are subsequences $\tilde{u}_{n}(x, s)$ $=\sum_{i=1}^{n} u_{i}(x) q_{i}^{(n)}(s)$ and $\sigma_{n(1)}(R)$ such that $\tilde{u}_{n}(x, s) \rightarrow \tilde{u}(x, s)$ weakly in $\dot{W}_{1,2}\left(G_{2 \pi}\right)$ and $\sigma_{n(1)}(R) \rightarrow \sigma_{1}(R)$ where $\int_{0}^{2 \pi} \int_{G}(\partial \tilde{u} / \partial s)^{2}=R$. Hence $\tilde{u}\left(x, \sigma_{1}^{-1}(R) t\right)$ is a $2 \pi \sigma_{1}(R)$ periodic weak solution of $(2)$, and as $R \rightarrow 0$, one finds $\sigma_{1}(R) \rightarrow 1 / \lambda_{1}$ provided $f(x, u)=o(u)$. Theorem 2 follows by replacing $n(1)$ by $n(i)\{i=2,3,4, \cdots\}$ in the above argument. The existence of the above limits is proven by using conditions $\left({ }^{*}\right)$ and (I.) to find a priori bounds on $\tilde{u}_{n}$ and its derivatives that are independent of $n$.

4. Extensions. (1) Let $A$ be a strongly elliptic linear selfadjoint operator of order $2 \mathrm{~m}$. Then the methods used to prove Theorems 1 and 2 can be applied to study the periodic solutions of the system

$$
\begin{aligned}
u_{t \iota}+(-1)^{m} A u+f\left(x, u, D u, \cdots, D^{2 m-2} u\right) & =0 & & \text { in } G \\
\left.D u\right|_{\partial G} & =0 & & |\alpha| \leqq m-1
\end{aligned}
$$

provided $f\left(x, u, \cdots, D^{2 m-2} u\right)$ satisfies suitable positivity and growth conditions and is derivable as an Euler-Lagrange expression of a functional $\int_{G} F\left(x, u, \cdots, D^{m-1} u\right)$. 


\section{BIBLIOGRAPHY}

1. M. S. Berger, On one parameter families of nonlinear operator equations, Bull. Amer. Math. Soc. 75 (1969), 456-459.

2. L. Cesari, Periodic solutions of nonlinear hyperbolic partial differential equations, Arch. Rational Mech. Anal. 20 (1965), 170-190.

3. P. H. Rabinowitz, Periodic solutions of nonlinear hyperbolic partial differential equations, Comm. Pure Appl. Math. 20 (1967), 145-205.

4. D. H. Sattinger, On Global solutions of nonlinear hyperbolic equations, Arch. Rational Mech. Anal. 30 (1968), 148-172.

5. $\mathrm{O}$. Vejvedo, Periodic solutions of a weakly nonlinear wave equation, Czechoslovak Math. J. 14 (1964), 341-382.

University of Minnesota, Minneapolis, Minnesota 55455, and

Belfer Graduate School, Yeshiva University, New York 10033. 